\title{
ChemComm
}

\section{Diastereomeric ratio determination by high sensitivity band-selective pure shift NMR spectroscopy $\dagger$}

Cite this: Chem. Commun., 2014 50,2512

Received 20th December 2013 Accepted 16th January 2014

DOI: $10.1039 /$ c3cc49659g

\author{
Ralph W. Adams, ${ }^{a}$ Liam Byrne, ${ }^{a}$ Péter Király, ${ }^{\text {ab }}$ Mohammadali Foroozandeh, ${ }^{a}$ \\ Liladhar Paudel, ${ }^{a}$ Mathias Nilsson, ${ }^{\text {ac }}$ Jonathan Clayden ${ }^{a}$ and Gareth A. Morris ${ }^{\star a}$
}

www.rsc.org/chemcomm

\begin{abstract}
An NMR method is reported that allows diastereomeric ratios to be determined even in crowded spectra or where chemical shift differences are small compared to multiplet widths. Band-selective pure shift NMR collapses multiplets to singlets, greatly improving spectral resolution while largely retaining, or even enhancing, signal-to-noise ratio.
\end{abstract}

The intrinsic chirality of many of the building blocks of nature requires chemists to create molecules with specific stereochemical configurations, in order to govern their interactions with naturally-occurring chemical and biological species. Substrate-directable chemical reactions, ${ }^{1}$ in which regio- and stereochemistry are controlled, are crucial for the synthesis of almost all complex natural products, and many pharmaceuticals. Measurement of the degree of stereochemical control is essential in choosing appropriate reactions and conditions, and in process optimisation. HPLC and NMR spectroscopy are used routinely to determine diastereomeric ratio (dr). However, in large molecules the presence of many overlapping signals and the small shift differences that are seen when chiral centres are well-separated can make determination of dr by NMR difficult, inaccurate, or impossible.

Much of the overlap in ${ }^{1} \mathrm{H}$ NMR spectra results from the multiplet structure caused by homonuclear scalar coupling. The recent development of 'pure shift' NMR methods allows broadband homodecoupling, collapsing multiplets to singlets and leaving a single peak for each chemically distinct site, dramatically improving spectral resolution. ${ }^{2-5}$ However, until

\footnotetext{
${ }^{a}$ School of Chemistry, University of Manchester, Oxford Road, Manchester, M13 9PL, UK. E-mail: g.a.morris@manchester.ac.uk; Tel: +44 (0)161 2754665

${ }^{b}$ Institute of Organic Chemistry, Hungarian Academy of Sciences, Pusztaszeri út 59-67, 1025 Budapest, Hungary

${ }^{c}$ Department of Food Science, University of Copenhagen, Rolighedsvej 30, 1958 Frederiksberg C, Denmark

$\dagger$ Electronic supplementary information (ESI) available: Details of experimental and processing parameters used for NMR experiments, pulse sequence for interferogram-based band-selective pure shift NMR, and structures of the diastereomers. Original data and parameter files are available from the authors on request. See DOI: 10.1039/c3ec49659g
}

very recently methods for $1 \mathrm{D}$ pure shift ${ }^{1} \mathrm{H}$ NMR have come with a significant penalty in signal-to-noise ratio. The two most successful classes of pure shift method, based respectively on BIRD (BIlinear Rotation Decoupling) ${ }^{4-7}$ and Zangger and Sterk's (ZS) spatially- and frequency-selective refocusing, ${ }^{2,8-11}$ generally cost one or two orders of magnitude in sensitivity if the whole spectrum is to be decoupled. In real-time acquisition experiments, however, it is possible to obtain simultaneous improvements in both sensitivity and resolution. ${ }^{7}$

Here we describe the application of band-selective pure shift NMR experiments, developed in this group and the groups of Parella and Bax, ${ }^{12-14}$ to the determination of dr. These experiments exploit - with much greater efficiency - a pulse sequence element demonstrated previously for decoupling the indirect dimension of a TOCSY or COSY experiment. ${ }^{15}$ The real-time method enhances both sensitivity and resolution, while the interferogram-based analogue provides optimum resolution but with a modest penalty in acquisition time. Reducing overlap between reporter signals, and between reporter and other signals, allows dr to be determined more easily and more accurately, whether by integration or by line shape analysis.

Pure shift NMR methods interleave periods of free signal evolution with J-refocusing elements. These reverse the effects of couplings (scalar or dipolar ${ }^{14}$ ), while leaving those of chemical shift unchanged. The band-selective decoupling (BSD) J-refocusing element used here is simply the ZS combination of a selective and a non-selective $180^{\circ}$ pulse, but with no gradient applied during the former. A pure shift free induction decay can be obtained either by alternating periods of free evolution and J-refocusing during data acquisition (real-time methods), or by constructing an equivalent decay from short chunks of free induction decay each measured using a single evolution time $t_{1}$ with a J-refocusing element at its centre (interferogram methods). In both cases the result of Fourier transformation is an NMR spectrum with a singlet at each chemical shift. The advantage of BSD methods is that all the available spins contribute to the required signal, rather than the small minority seen in BIRD and ZS experiments. 
In the determination of $\mathrm{dr}$ the chemist is generally interested only in the integral ratios of a few diagnostic signals, typically those of protons lying close to the stereogenic centres of interest; the rest of the spectrum is superfluous. It is therefore much more efficient to acquire a BSD pure shift NMR spectrum, containing only the spectral region of interest with couplings to spins outside the region suppressed, rather than measuring a complete pure shift spectrum at a large penalty in the measurement time required.

Conventional selective (or band-selective) homonuclear decoupling is performed by irradiating a single ${ }^{1} \mathrm{H}$ multiplet during acquisition using coherent irradiation ${ }^{16}$ (or a shaped RF pulse $^{17}$ ) in time-shared mode. ${ }^{18,19}$ Band-selective time-shared experiments have to target all the spins to which the spins of interest are coupled, are particularly challenging to set up because of Bloch-Siegert and off-resonance effects, and are relatively little used. Pure shift BSD experiments, in contrast, target only the spins of interest and are much simpler.

The real-time BSD pure shift pulse sequence used, which has at its core an analogue of the most commonly-used variant of the ZS pulse sequence, ${ }^{2,8}$ is shown in Fig. 1. The magnetic field gradient usually applied during the ZS selective refocusing pulse is removed, to give selective refocusing of a defined band of chemical shifts. Equivalent methods have been described recently for band-selective pure shift NMR of peptides. ${ }^{13,14}$ The $180^{\circ}$ refocusing pulse is tailored to affect a band of chemical shifts containing only one multiplet from each spin system, so that all couplings are refocused and only singlets result in the final spectrum.

The real-time sequence shown refocuses the effects of $\mathrm{J}$ between short chunks of data acquisition. This prevents the effects of J-evolution from building up, so only the effects of the chemical shift are observed in the FID and resultant spectrum. ${ }^{5,7,10}$ In the analogous interferogram sequence (see ESI, $\dagger$ Fig. S1), each chunk of data is acquired in a separate experiment as a chemical shift

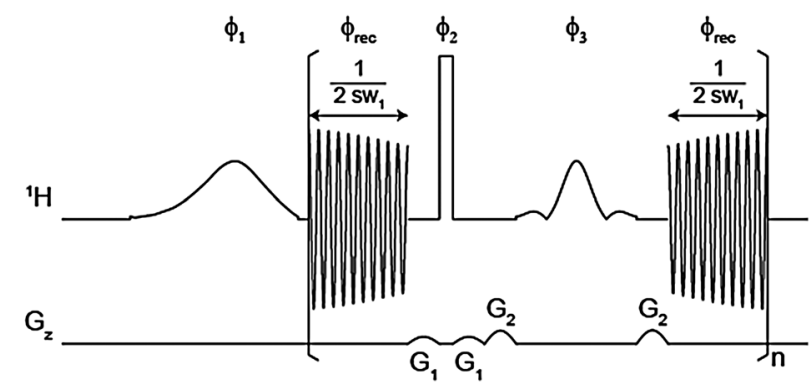

Fig. 1 Real time pure shift sequence using a $90^{\circ}$ selective excitation pulse (eBURP) with phase $\phi_{1}$, and a J-refocusing element comprising a broadband $180^{\circ}$ pulse with phase $\phi_{2}$ and a band-selective (rSNOB) $180^{\circ}$ refocusing pulse with phase $\phi_{3}$. Data points are collected using a looped, windowed acquisition with phase $\phi_{\text {rec }}$. The J-refocusing and acquisition loop is repeated $n$ times until the required number of data points have been collected. Phase cycle: $\phi_{1}=x,-x,-x, x, y,-y,-y, y ; \phi_{2}=x,-x$; $\phi_{3}=-x, x ; \phi_{\mathrm{rec}}=\phi_{1}$. The duration $1 / \mathrm{sw}_{1}$ of the acquisition chunks depends on the balance required between resolution and spectral purity; a good compromise is an $\mathrm{sw}_{1}$ roughly twice the maximum multiplet width in the normal spectrum. evolution time $t_{1}$ is incremented, just as in a two-dimensional NMR experiment. ${ }^{4,8}$ The J-evolution is prefocused so that at the centre of each chunk only chemical shift terms have accumulated. The real-time method of Fig. 1 requires approximately the same experiment time as a conventional measurement, and offers similar or better sensitivity. However, relaxation during the successive selective pulses does slightly reduce the resolution advantage, which is not the case for the interferogram method. The disparity depends on the bandwidth; the wider the region of interest, the shorter the selective pulses and the smaller the extra relaxation contribution. Thus while the real-time experiment is preferable for most purposes, the interferogram experiment comes into its own where the region of interest is narrow and where resolution is paramount. In both experiments relaxation during the pulse sequence slightly affects the signal integrals measured, but such effects would only become a problem in systems with relaxation too fast for pure shift methods to offer much resolution advantage.

Where signal resolution remains a problem even after the use of pure shift methods, a chemical additive may be introduced that can separate signals. The traditional additive would be a chiral lanthanide shift reagent ${ }^{20}$ but other chiral species can also provide good resolution of diastereomer, ${ }^{21}$ and even enantiomer, signals. Conversely, if NMR multiplet signals are not fully resolved after addition of additives the subsequent use of band-selective pure shift NMR may allow their separation and quantification. ${ }^{22}$

The benefits of band-selective pure shift NMR for quantifying $\mathrm{dr}$ are illustrated in Fig. 2 for the flavanone glycoside hesperidin. Hesperidin exists naturally as a mixture of two diastereomers (see ESI, $\dagger$ Fig. S2) in many citrus fruits. The dr is dependent on the species of citrus, the ripeness of the fruit,

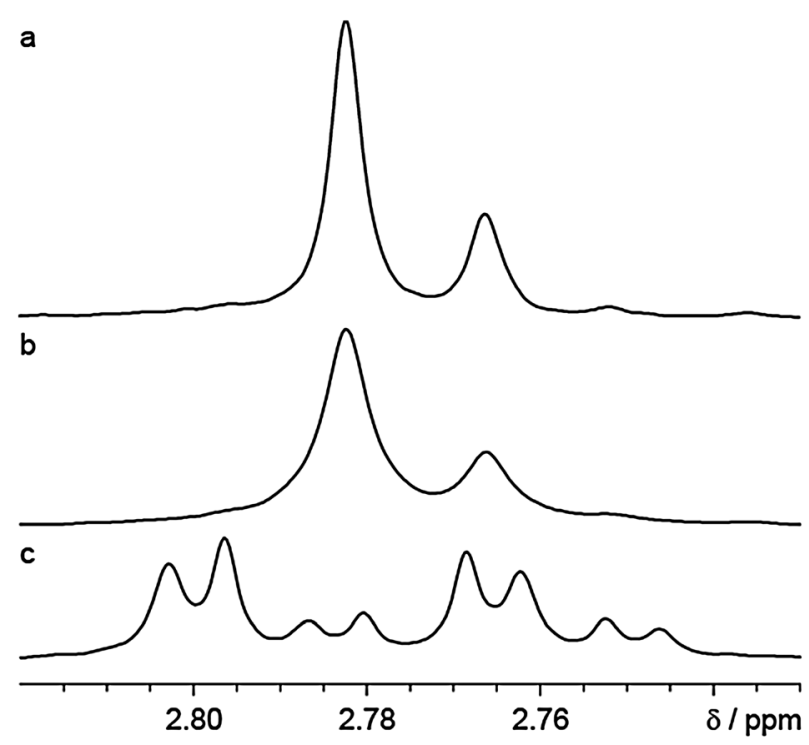

Fig. $2500 \mathrm{MHz}{ }^{1} \mathrm{H}$ NMR spectra showing $d r$ reporter signals for a mixture of hesperidin diastereomers in $\mathrm{d}_{6}$-DMSO acquired using (a) interferogrambased and (b) real-time band-selective pure shift experiments, and (c) standard acquisition. Experimental and processing parameters were consistent between the experiments; full details are given in the ESI. $\dagger$ 
a

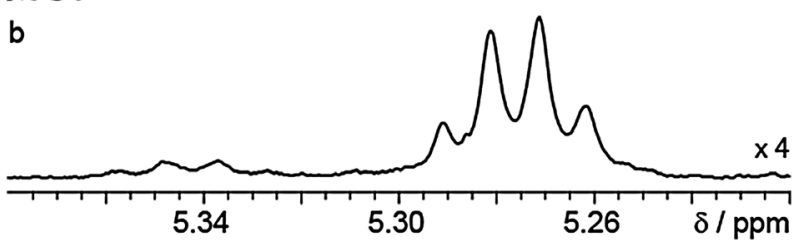

Fig. $3800 \mathrm{MHz}{ }^{1} \mathrm{H}$ NMR spectra in $\mathrm{d}_{8}$-THF of the diastereomeric oligomeric products of an enamide addition reaction resulting in 1,61-asymmetric induction (see $\mathrm{ESI} \dagger$ and ref. 12). The multiplet structure in the standard proton experiment (b) is collapsed in the interferogrambased pure shift NMR experiment (a) where signal intensity also increases. Experimental and processing parameters are consistent for the spectra and are given in the ESI. $\dagger$

and the temperature at which extraction occurred. ${ }^{23}$ Two sets of signals, one from each diastereomer, occur in the ${ }^{1} \mathrm{H}$ NMR spectrum, at the chemical shifts shown in Fig. 2 . The signals in the normal spectrum (Fig. 2c) are split into overlapping doublets of doublets, making quantification of $\mathrm{dr}$ difficult. Using the bandselective pure shift method of Fig. 1 collapses the multiplets, making integration and hence determination of dr straightforward.

In a second example, the crude product mixture of a remotely diastereoselective reaction entailing foldamer-mediated 1,61 stereocontrol $^{12}$ contained two diastereomers (epimeric at the benzylic chiral centre, see ESI, $\dagger$ Fig. S3) and several minor impurities. The diastereoselectivity of the reaction, in which a facially selective attack on an acyliminium ion was induced through an extended achiral helix, could not be determined confidently by standard ${ }^{1} \mathrm{H}$ NMR methods because of signal overlap. Changing solvent from $\mathrm{d}_{4}$-MeOD to $\mathrm{d}_{8}$-THF allowed the two diastereomer signals to be distinguished, only to reveal underlying impurity signals (Fig. 3b). The combination of low available concentration and a single reporter region made the band-selective pure shift method particularly attractive here. Even at $800 \mathrm{MHz}$, using the higher resolution interferogrambased experiment (see ESI, $\dagger$ Fig. S1), a shoulder is visible on the main diastereomer signal. Lorentzian line shape fitting rather than direct integration was therefore used, giving a dr of $12: 88$ rather than the drs of $8: 92$ and $7: 93$ obtained by integration of the pure shift and conventional ${ }^{1} \mathrm{H}$ spectra respectively. The $\mathrm{dr}$ obtained by lineshape fitting of the pure shift NMR data was consistent with the expected degree of local asymmetry induced by the achiral helix mediating the remote asymmetric induction.
NMR is a valuable tool for measurement of diastereomeric ratios, whether of diastereomeric materials or of diastereomers made from chiral substrates using chiral derivatising or chemical shift reagents, but resolution and sensitivity are critical. Band-selective pure shift NMR methods both simplify the determination of diastereomeric ratio and improve its reliability.

This work was funded by the EPSRC (EP/I007989), the European Research Council (Advanced Grant ROCOCO), and by a UR Grant from the Agilent Technologies Foundation. P.K. thanks EMBO for a Short-Term Fellowship (ASTF 513-2012).

\section{Notes and references}

1 A. H. Hoveyda, D. A. Evans and G. C. Fu, Chem. Rev., 1993, 93, 1307-1370.

2 K. Zangger and H. Sterk, J. Magn. Reson., 1997, 124, 486-489.

3 M. Nilsson and G. A. Morris, Chem. Commun., 2007, 933-935.

4 J. A. Aguilar, M. Nilsson and G. A. Morris, Angew. Chem., Int. Ed., 2011, 50, 9716-9717.

5 A. Lupulescu, G. L. Olsen and L. Frydman, J. Magn. Reson., 2012, 218, 141-146.

6 J. R. Garbow, D. P. Weitekamp and A. Pines, Chem. Phys. Lett., 1982, 93, 504-509.

7 L. Paudel, R. W. Adams, P. Király, J. A. Aguilar, M. Foroozandeh, M. J. Cliff, M. Nilsson, P. Sándor, J. P. Waltho and G. A. Morris, Angew. Chem., Int. Ed., 2013, 52, 11616-11619.

8 J. A. Aguilar, S. Faulkner, M. Nilsson and G. A. Morris, Angew. Chem., Int. Ed., 2010, 49, 3901-3903.

9 N. Giraud, L. Béguin, J. Courtieu and D. Merlet, Angew. Chem., Int. Ed., 2010, 49, 3481-3484.

10 N. H. Meyer and K. Zangger, Angew. Chem., Int. Ed., 2013, 52, 7143-7146.

11 N. Giraud, M. Joos, J. Courtieu and D. Merlet, Magn. Reson. Chem., 2009, 47, 300-306.

12 L. Byrne, J. Solà, T. Boddaert, T. Marcelli, R. W. Adams, G. A. Morris and J. Clayden, Angew. Chem., Int. Ed., 2014, 126, 155-159.

13 L. Castañar, P. Nolis, A. Virgili and T. Parella, Chem.-Eur. J., 2013, 19, 17283-17286.

14 J. Ying, J. Roche and A. Bax, J. Magn. Reson., 2013, DOI: 10.1016/ j.jmr.2013.11.006.

15 V. V. Krishnamurthy, Magn. Reson. Chem., 1997, 35, 9-12; N. Nath, D. Kumari and N. Suryaprakash, Chem. Phys. Lett., 2011, 508, 149-154.

16 P. Jesson, P. Meakin and G. J. Kneissel, J. Am. Chem. Soc., 1973, 95, 618-620.

17 A. P. D. M. Espindola, R. Crouch, J. R. DeBergh, J. M. Ready and J. B. MacMillan, J. Am. Chem. Soc., 2009, 131, 15994-15995.

18 J. Huth, N. D. Kurur and G. Bodenhausen, J. Magn. Reson., Ser. A, 1996, 118, 286-290.

19 B. Baishya, T. F. Segawa and G. Bodenhausen, J. Magn. Reson., 2011, 211, 240-242.

20 A. K. Rogerson, J. A. Aguilar, M. Nilsson and G. A. Morris, Chem. Commun., 2011, 47, 7063-7064.

21 R. W. Adams, J. A. Aguilar, J. Cassani, G. A. Morris and M. Nilsson, Org. Biomol. Chem., 2011, 9, 7062-7064.

22 D. Parker, Chem. Rev., 1991, 91, 1441-1457.

23 N. Uchiyama, I. H. Kim, N. Kawahara and Y. Goda, Chirality, 2005, 17, 373-377. 